\title{
Fuzzy Goal Programming Approach for Integrating Production and Distribution Problem in Milk Supply Chain
}

\author{
Achraf Touil ${ }^{\mathrm{a}}$, Abdelwahed Echchatbi and Abdelkebir Charkaoui \\ Laboratory of Engineering, of Industrial Management and Innovation, FST, BP: 577, Settat, Morocco
}

\begin{abstract}
In this paper, a bi-objective mixed integer programming model is proposed to deal with the productiondistribution problem found in a dairy company in Morocco. The supply chain containing three echelons: multi-sites, multi-distribution centers and multi-customers. The model seeks to integrate two conflicting simultaneous objectives: maximizing benefit by considering the shelf life of products and the total cost (quantitative objective), including production, storage, and distribution, as well as maximizing the service level (qualitative objective), which relates to providing satisfactory services to customers. This is subject to several technological constraints that typically arise in the dairy industry, such as sequence-dependent changeover time, machine speed and storage capacity. Due to imprecise aspiration levels of goals, an interactive approach is proposed based on fuzzy goal additive variants to find an efficient compromise solution. Numerical results are reported to demonstrate the efficiency and applicability of the proposed model.
\end{abstract}

\section{Introduction}

The food industry is growing in Morocco in recent years, mainly the dairy industry, which represents an important activity - one that contributes to the development of the economy by an agricultural GDP of $30 \%$, and a coverage rate of approximately $90 \%$ of the national demand for milk and dairy products. However, the sector is characterized by several challenges, namely high market competition, demand remaining below requirements recommended by nutritional standards, the specific characteristics of the raw material (perishability), and diversification of ranges of products. Due to of these factors, the enterprises investigate the possibility to turn these challenges to a competitive advantage while continuously improving their operation. One of the key activities to achieve this goal is by an efficient integrate of production and distribution planning.

In supply chain management, the production and distribution operations can be decoupled if there is a sufficient buffer between them. Related to the dairy SC, poor integration within a network may consist of excessive inventories at the manufacturers' warehouses. Regarding the perishability characterized, the finished products should be kept refrigerated at a controlled temperature that results in a significant cost of storage, which may influence efficiencies. Therefore, we consider a model to integrate the production and distribution problem.

In academic literature, a considerable number of studies have examined the importance of the productiondistribution problem in supply chain management. A comprehensive review on integrating productiondistribution problems has been presented by [1-3].

Related to the dairy supply chain, a comprehensive study is proposed to deal with the production-distribution problem. However, milk production is a significant activity in the dairy industry, and the literature regarding this subject is very scarce. Nonetheless, some research has been conducted considering other dairy products, such as yogurt and ice cream. The studies related to milk supply chain management can be classified according to the problem characteristics and the proposed solution methodologies. The problem characteristic refers to decision levels, supply chain processes, product type, production process, perishability, working time and changeovers. The proposed solution methods vary from MILP and CP to stochastic programming (SP) and simulation (SM). A comprehensive review of quantitative models for the dairy industry was discussed by [4] and [5].

In this paper, we study a multi-product, multi-echelon milk supply chain containing three echelons: multi-sites, multi-distribution centers and multi-customers, which is inspired by the formulation previously considered in [4]. The problem lies in assigning the products to the processing units and determining the inventory level of each product at production sites and distributions centers, subject to sets of constraints that must be satisfied, such as capacity constraints, production, and storage. The model seeks to simultaneously incorporate two objectives: the first objective refers to maximizing the benefit by considering the shelf life of products [6] and the total costs, including production, storage, distribution and back

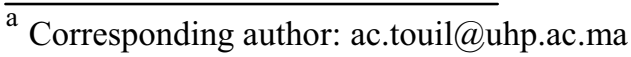


order, while the second objective is relevant to maximizing the service level. In addition, the model takes into account the transport lead time between different echelons of the supply chain. Because the imprecise aspiration level of objectives, a fuzzy bi-objective mixed integer model is proposed to deal with this issue. The main contributions of this paper can be summarized as follows: First, we propose a MILP formulation for the production-distribution problem encountered by a milk company. Second, the maximization and minimization goals for each objective are given and are converted to fuzzy goals using fuzzy membership values. Each fuzzy goal is assigned with a weight and all fuzzy goals are combined together to frame the Weighted Additive Fuzzy Goal model. Taking the importance of each individual objective into consideration, the weight for each objective is given.

The remainder of this paper is organized as follows. The proposed bi-objective mixed integer programming model is elaborated in Section 2. The proposed solution method is given in Section 3. Computational experiments are reported in Section 4 and finally, the concluding remarks and some directions for future research are given in Section 5.

\section{Bi-objective mathematical model}

\subsection{Problem statement}

The following considerations further define and delimit the problem:

- The dairy supply chain consists of several production sites and several DCs that deliver the final products to various customers.

- Each production site comprises several not necessarily identical parallel processing units.

- Due to restrictions on hygiene and security in the dairy industry, a setup operation between products is required, which results in setup time and cost.

- The scheduling horizon is supposed to be five days. The time horizon of short-term planning for dairy manufacturing is usually one week [7].

- The time for the quality control process is considered in the model. It is not allowed that the products are delivered before they complete the required time for the quality control process. A quarantine time is defined as a precondition for achievement of the desired sensory qualities [6].

- Freshness, which plays a significant part in the competitiveness, is taken into account for profitability. The objective function aims at maximizing the contribution margin given a shelf life-dependent pricing component, as stated in the model developed by [6].

- The available working time for the processing units is defined according to the shifts. Overtime production is considered on the third shift of every working day and under an environment of heavy demand, and if necessary, the production day can proceed in weekdays that are defined as overtime. The cleaning and sterilization operations are ensured on the third shift of each production day.
- Customer demand for each product type is daily collected from DCs.

- The processing unit capacity should not exceed a maximum capacity.

- The capacity of storage at sites and DCs is limited. In this model, we considered the safety stock level and the storage capacity related to each type of product to each site and each DC. Also, the total storage capacity of both sites and centers.

- The transport parameters, such as transport costs and transport lead times, are taken into consideration.

The key decisions of the model are:

- The production amount that is produced by each processing unit and in each period.

- The assignment of products to each unit, and in each period.

- The dispatched amount between (Sites-DCs) and (DCs-Customers) in each demand period.

- The inventory level of each product at each site and center at the end of each period.

- Sales and demand backlog of each product in each demand period.

- Service level desired by each customer.

\subsection{Model formulation}

$$
\begin{aligned}
& \text { Indices } \mid \text { Sets } \\
& t \in T: \text { Planning periods (days). } \\
& n \in N: \text { Demand periods (days). } \\
& p, k, l, e \in P: \text { Finished products (products). } \\
& a \in A: \text { Distribution centers (DCs). } \\
& s \in S: \text { Production sites (sites). } \\
& j \in J: \text { Processing units (units). } \\
& c \in C: \text { Customers. }
\end{aligned}
$$

$J_{p}:$ Units $\mathrm{j}$ that can processed product $\mathrm{p}$.

$J_{s}:$ Units $\mathrm{j}$ that are installed on site $s$.

$S_{a}:$ Sites s that can supply center $a$.

$A_{s}:$ DCs $a$ that are supplied by site $s$

$A_{c}$ : DCs $a$ that can supply customer c

$C_{a}$ : Customers c that are provided by DC a.

\section{Parameters}

$D_{c, p, n}$ : Demand of product $\mathrm{p}$ required by customer c on demand day $\mathrm{d}$.

Benefit $_{p}$ : Maximum benefit for meeting the highest shelf life of product $\mathrm{p}$,

$C r_{p}$ : Critical rate for the shelf life of product $\mathrm{p}$ required by customers.

$s h l_{p}$ : Shelf life of product $\mathrm{p}$, (day).

$q q t_{p}$ : Quarantine time needed to control product quality, (day).

rate $_{p, s, j}$ : Processing rate of product $\mathrm{p}$ on unit $\mathrm{j}$ at site $\mathrm{s}$.

$\left[\right.$ Lot $\left._{p}, \overline{\operatorname{Lot}_{p}}\right]$ : Minimum and maximum lot size of product $\mathrm{p}$. 
$V P C_{p, s, j, t}$ : Variable production cost of one unit of product $\mathrm{p}$ in unit $\mathrm{j}$ of the site $\mathrm{s}$ in period $\mathrm{t}$ (including labor and utilities cost).

$I H C S_{p, s, t}$ : Inventory holding cost for one unit of product $\mathrm{p}$ at site $\mathrm{s}$ in period $\mathrm{t}$.

$S S S_{p, s, t}$ : Safety stock of product $\mathrm{p}$ at site $\mathrm{s}$ in period $\mathrm{t}$.

$S C S_{p, s}$ : Storage capacity associated with product $\mathrm{p}$ at site s.

$\operatorname{TSCS}_{s}$ : Total storage capacity of site s.

$\phi_{p, k, s, j, t}$ : Changeover time between product $\mathrm{p}$ and product $\mathrm{k}$ on unit $\mathrm{j}$ of site $\mathrm{s}$ in period $\mathrm{t}$.

$\chi_{p, k, s, j, t}$ : Changeover cost between product $\mathrm{p}$ and product $\mathrm{k}$ on unit $\mathrm{j}$ of site $\mathrm{s}$ in period $\mathrm{t}$.

$O T C_{s, j, t}$ : Overtime cost associated with unit $\mathrm{j}$ of site $\mathrm{s}$ in period $\mathrm{t}$.

$I H C D_{p, s, t}$ : Inventory holding cost for one unit of product $\mathrm{p}$ at center in period $\mathrm{t}$.

$S S A_{p, a, t}$ : Safety stock of product $\mathrm{p}$ at center in period $\mathrm{t}$.

$S C A_{p, a}$ : Storage capacity associated with product $\mathrm{p}$ at center a.

$T S C A_{a}$ : Total storage capacity of center a.

$T C D_{s, a}$ : Transport cost from site s and center a.

$T C C_{a, c}$ : Transport cost from center $a$ and customer c.

$T L T 1_{s, a}$ : Transport lead-time between site $s$ and center

a.

TLT2 $2_{a, c}$ : Transport lead-time between center $a$ and customer c.

$B C_{p, c, t}$ : Backorder cost per unit of product $\mathrm{p}$ from customer $\mathrm{c}$ at the end of period $\mathrm{t}$.

$\operatorname{Cap}_{S, j}^{\max }$ : Maximum production capacity of unit $\mathrm{j}$ at site S.

$R t_{s, j, t}$ : Regular in use shift of unit $\mathrm{j}$ at site $\mathrm{s}$ in period $\mathrm{t}$.

M: Extremely large number.

\section{Variables}

Binary variables

$Y_{p, s, j, t}=1$ if product $\mathrm{p}$ is assigned to a unit $j \in J_{p}$ at site $s$ in period t.

$Z_{p, k, s, j, t}=1$ if product $\mathrm{p}$ is processed before product $\mathrm{k}$ when both are assigned to the same unit $j \in J_{p}$ at site $\mathrm{s}$ in period $\mathrm{t}$.

\section{Continuous variables}

$Q P S_{p, s, j, t, n}$ : Amount of product p produced by unit $\mathrm{j}$ at site $s$ in period $\mathrm{t}$ for demand period $\mathrm{n}$.

$Q T D_{s, a, p, t, n}$ : Amount of product $\mathrm{p}$ sent from site $s$ to center $a$ in period $\mathrm{t}$ for demand period $\mathrm{n}$.

$Q T C_{a, c, p, t, n}$ : Amount of product p sent from center $a$ to customer $\mathrm{c}$ in period $\mathrm{t}$ for demand period $\mathrm{n}$.

$I S_{s, p, t}$ : Inventory level of product $\mathrm{p}$ at site $s$ at end of period $\mathrm{t}$.
$I D_{a, p, t}$ : Inventory level of product $\mathrm{p}$ at center $a$ at end of period $\mathrm{t}$.

$B P_{c, p, n}$ : Amount of product $\mathrm{p}$ backordered by customer $\mathrm{c}$ on demand period $\mathrm{n}$.

$S L_{c, p, n}$ : Desired service level at customer c related to product $\mathrm{p}$ in period $\mathrm{t}$ for demand period $\mathrm{n}$.

$O T_{s, j, t}$ : Overtime on unit $\mathrm{j}$ at site $\mathrm{s}$ in period $\mathrm{t}$.

$P T_{p, s, j, t}$ : Processing time of product $\mathrm{p}$ on unit $\mathrm{j}$ at site $s$ in period $\mathrm{t}$.

$S T_{p, s, j, t}$ : Starting time of product $\mathrm{p}$ on unit $\mathrm{j}$ at site $S$ in period $\mathrm{t}$.

$C T_{p, s, j, t}$ : Finishing time of product $\mathrm{p}$ on unit $\mathrm{j}$ at site $s$ in period $\mathrm{t}$.

$C_{s, j, t}$ : Finishing time of the last product on unit $\mathrm{j}$ at site $s$ in period $\mathrm{t}$.

\section{Objective function}

Minimization of total cost included (ProductionStorage-Distribution)

$B=\sum_{s} \sum_{p} \sum_{j \in J_{p} \cap J_{k}} \sum_{t} \sum_{d} B_{p} * \frac{\left(1-C r_{p}\right)^{*} S h l_{p}-(d-t)}{\left(1-C r_{p}\right)^{*} S h l_{p}}$

${ }^{*} Q P S_{p, s, j, t, n}$

$T C=\sum_{s} \sum_{p} \sum_{j \in J_{s} \cap J_{p}} \sum_{t} \sum_{d} V P C_{p, s, j, t} * Q P S_{p, s, j, t, n}$

$+\sum_{s} \sum_{j} \sum_{t} O T C_{s, j, t} * O T_{s, j, t}$

$+\sum_{s} \sum_{p} \sum_{k \neq p} \sum_{j \in J_{s} \cap J_{p} \cap J_{k}} \sum_{t} \phi_{p, k, s, j, t} * \chi_{p, k, s, j, t}$

$+\sum_{s} \sum_{p} \sum_{t} I S_{s, p, t} * \mathrm{IHCS}_{s, p, t}$

$+\sum_{s} \sum_{a \in A_{s}} \sum_{t} \sum_{n} Q T D_{s, a, p, t, n}^{*} T C D_{s, a}$

$+\sum_{a} \sum_{p} \sum_{t} I D_{c, p, t} * I H C D_{c, p, t}$

$+\sum_{a} \sum_{c \in C_{a}} \sum_{t} \sum_{n} Q T C_{a, c, p, t, n}{ }^{*} T C C_{a, c}$

$+\sum_{c} \sum_{p} \sum_{n} B P_{c, p, n}{ }^{*} B C_{c, p, n}$

$$
\text { Max } Z_{\text {Profit }}=B-T C
$$

The first component of the objective function (1) seeks to maximize benefit by considering the shelf life of products. It is assumed that the manufacturer yields a financial benefit if the products have a longer residual shelf life when being delivered [6]. The other component describes the setup, production, storage, distribution and backorder costs, respectively.

\section{Maximization of service level}

The last objective function aims to maximize the service level related to each product by the customer: 


$$
S L_{c, p, n}=1-\frac{\sum_{n} B P_{c, p, n}}{\sum_{n} D_{c, p, n}}
$$

\section{Constraints}

Constraint (3) ensures the lower and upper bounds on lot product sizes.

$$
\begin{gathered}
L_{\operatorname{Lot} p}^{* Y_{p, s, j, t}} \leq \sum_{n=t} Q P S_{p, s, j, t, n} \leq \overline{\operatorname{Lot}_{p}} Y_{p, s, j, t} \\
\forall s, j, p, t
\end{gathered}
$$

The processing time of product $p$ on unit $j$ that equals the produced amount divided by the rate of the same unit is ensured by Constraint (4).

$$
\begin{gathered}
P T_{p, s, j, t}=\frac{\sum_{n} Q P S_{p, s, j, t, n}}{\text { rate }_{p, s, j}} \\
\forall s, p, j \in J_{p} \cap J_{s}, t
\end{gathered}
$$

The finishing time of product $\mathrm{p}$ on unit $\mathrm{j}$ at site $\mathrm{s}$ in period $t$ is equal to the sum of its corresponding starting time and processing time on the same unit and is described by Constraint (5).

$$
\begin{gathered}
C T_{p, s, j, t}=S T_{p, s, j, t}+P T_{p, s, j, t} \\
\forall s, p, j \in J_{p} \cap J_{s}, t
\end{gathered}
$$

Constraint (6) ensures that the starting time of the product that directly follows another product $\mathrm{k}$ on a processing unit $\mathrm{j}$ at production site $\mathrm{s}$ in period $\mathrm{t}$ (i.e., $\left.Z_{p, k, s, j, t}=1\right)$ should be greater than the completion time of product $\mathrm{k}$ ( $\left.C T_{p, s, j, t}\right)$, plus the necessary changeover time $\phi_{p, k, s, j, t}$ between those products. Similarly, Constraint (7) describes the opposite case.

$$
\begin{gathered}
C T_{k, s, j, t} \geq C T_{p, s, j, t}+\phi_{p, k, s, j, t}-M^{*} Z_{p, k, s, j, t}- \\
M^{*}\left(1-Y_{p, s, j, t}-Y_{k, s, j, t}\right) \\
\forall s, p, j \in J_{p} \cap J_{k} \cap J_{s}, t \\
C T_{p, s, j, t} \geq C T_{k, s, j, t}+\phi_{k, p, s, j, t}-M^{*}\left(1-Z_{p, k, s, j, t}\right)- \\
M^{*}\left(1-Y_{p, s, j, t}-Y_{k, s, j, t}\right) \\
\forall s, p, j \in J_{p} \cap J_{k} \cap J_{s}, t
\end{gathered}
$$

Constraints (8-10) describe the product sequencing and timing. Constraints (8) and (9) ensure that if a product $\mathrm{p}$ is assigned to processing unit $\mathrm{j}$ at production site $\mathrm{s}$ in period $\mathrm{t}$ (i.e., $Y_{p, s, j, t}=1$ ) then at most one product $\mathrm{k}$ is processed before and after product $\mathrm{p}$, respectively. According to Constraint (10), the total number of active sequencing binary variables $Z_{p, k, s, j, t}$ should be less than the total number of active allocation binary variables $Y_{p, s, j, t}$ minus 1 in a processing unit $\mathrm{j}$ at production site $\mathrm{s}$ in period $\mathrm{t}$.

$$
\begin{gathered}
\sum_{k \in P, k \neq p} Z_{k, p, s, j, t} \leq Y p, s, j, t \\
\forall s, p, j \in J_{p} \cap J_{k} \cap J_{s}, t \\
\sum_{k \in P, k \neq p} Z_{p, k, s, j, t} \leq Y p, s, j, t \\
\forall s, p, j \in J_{p} \cap J_{k} \cap J_{s}, t \\
\sum_{k \in P, k \neq p} Z p, k, s, j, t \leq Y p, s, j, t \\
\forall s, p, j \in J_{p} \cap J_{k} \cap J_{s}, t
\end{gathered}
$$

Constraint (11) ensures the total processing time of the last product on unit $\mathrm{j}$ at site $\mathrm{s}$ in period $\mathrm{t}$.

$$
C_{s, j, t}=C T_{P, s, j, t}, \forall s, p, j \in J_{s}, t
$$

The constraint (12) ensures that the total processing time will not exceed the maximum available time for the same unit in period $t$.

$$
C_{s, j, t} \leq \operatorname{Cap}_{s, j}^{\max } \forall s, p, j \in J_{s}, t
$$

The overtime on unit $\mathrm{j}$ at site $\mathrm{s}$ in period $\mathrm{t}$ is computed according to Constraint (13).

$$
C_{s, j, t}=O T_{s, j, t}+R t_{s, j, t}, \forall s, p, j \in J_{s}, t
$$

Constraint (14) ensures that to meet the demand for demand day $\mathrm{d}$, the products should be produced within a window of time according to quarantine time and shelf life. The quarantine time is required for the achievement of the desired level of quality. The product cannot be delivered before a quarantine time has reached $n-t \geq q q t_{p}$. The shelf life lost (d-t), may not exceed a threshold provided by the customer $(n-t) \leq\left(1-C r_{p}\right) * S h l_{p}$. [6]. Otherwise, it should be noticed that $Q_{T}=0$.

$$
Q_{T}=\sum_{t} \sum_{n} \sum_{j \in J_{s}} Q P S_{p, s, j, t, n}, \forall s, p, t
$$

where $n=1:(n-t) \geq q q t_{p} \wedge(n-t) \leq\left(1-c r_{p}\right) * s h l_{p}$

The inventory of product $\mathrm{p}$ in production site $\mathrm{s}$ at the end of period $t$ is equal to the inventory of that product in the previous period, plus the total production quantity of product $\mathrm{p}$ at site $\mathrm{s}$ in period $\mathrm{t}$, minus the amount of product $\mathrm{p}$ transported from site $\mathrm{s}$ to center $a \in A_{S}$ in period $t$, is ensured by Constraint (15)

$$
I S_{s, p, t}=I S_{s, p, t-1}+Q_{T}-\sum_{a \in A} Q T D_{p, s, a, t-T L T 1, n}
$$




$$
\forall s, p, t
$$

Constraints (16-21) ensure the safety stocks, productdependent storage limitation, and the total capacity for product $\mathrm{p}$ at site $\mathrm{s}$ and distribution center, respectively.

$$
\begin{gathered}
I S_{S, p, t} \geq S S S_{p, s, t}, \forall s, p, t \\
I S_{S, p, t} \leq S C S_{p, s}, \forall s, p, t \\
\sum_{p} I S_{s, p, t} \leq T S C S_{s}, \forall s, p, t \\
I D_{a, p, t} \geq S S A_{p, a, t}, \forall a, p, t \\
I D_{a, p, t} \leq S C A_{p, a}, \forall a, p, t \\
\sum_{p} I D_{c, p, t} \leq T S C A_{a}, \forall a, p, t
\end{gathered}
$$

Constraint (22) describes the quantity of product $\mathrm{p}$ transported from center $a$ to customer $a$ in period t to adequately meet the demand period $\mathrm{d}$.

$$
\sum_{a \in A_{c}} Q T C_{p, a, c, t, n}=D_{c, p, n} \forall c, p, t, n
$$

Constraint (23) guarantees that the inventory level of product $\mathrm{p}$ at the end of period $\mathrm{t}$ must be equal to the amount entering from site $s \in S_{a}$, minus the amount dispatched to customers $a \in A_{c}$

$$
\begin{aligned}
& I D_{a, p, t}=I D_{a, p, t-1}+\sum_{s \in S} Q T D_{p, s, a, t, n}-\sum_{c \in C_{\text {so }}} Q T C_{p, a, c, t-T L T 2, n} \\
& \forall p, t, n
\end{aligned}
$$

The backlog level of product $\mathrm{p}$ incurred by client $\mathrm{c}$ for demand period $d$ equals the backlog level of that product in the previous period, plus the total demand of the product by client during that period, minus the total amount provided by the distribution center $a \in A_{\mathcal{C}}$, is shown by Constraint (24).

$$
\begin{gathered}
B P_{c, p, t}=B P_{c, p, t-1}+D_{c, p, t}-\sum_{a \in A_{c}} Q T C_{a, c, p, t, n} \\
\forall p, t, n
\end{gathered}
$$

\section{Solution approach}

Fuzzy linear programming was proposed by [8], and has been typically used to solve fuzzy multi-objective programming problems by converting such problems into the single objective formulation. A modified fuzzy linear programming version called WAFGP was proposed by [9]. In this method, each objective function $\left(Z_{k}\right)$ of a multi-objective optimization problem is resolved to obtain its minimum $\left(Z_{k}^{\min }\right)$ and maximum $\left(Z_{k}^{\max }\right)$ goals, individually satisfying all the constraints. All individual objectives are converted into fuzzy goals using their minimum and maximum goals, respectively [10].

$$
\begin{gathered}
\mu_{Z_{k}}=\left\{\begin{array}{l}
1 ; \quad Z_{k}(x) \prec Z_{k}^{\min } \\
\frac{Z_{k}^{\max }-Z_{k}(x)}{Z_{k}^{\max }-Z_{k}^{\min }} ; \quad Z_{k}^{\min } \prec Z_{k}(x) \prec Z_{k}^{\max } \quad(E q .1) \\
0 ; \quad Z_{k}(x) \succ Z_{k}^{\max }
\end{array}\right. \\
\mu_{Z_{l}}=\left\{\begin{array}{c}
0=1,2, \ldots, p \\
\frac{Z_{l}(x)-Z_{l}^{\min }}{Z_{l}^{\max }-Z_{l}^{\min }} \quad ; \quad Z_{l}^{\min } \prec Z_{l}(x) \prec Z_{l}^{\max } \quad(E q .2) \\
1 \quad Z_{l}(x) \succ Z_{l}^{\max } \\
l=p+1, p+2, \ldots, q
\end{array}\right.
\end{gathered}
$$

Fuzzy goals (FG) are combined, and the WAFGP model is formulated as shown in Eq.1 subjected to constraints as in Eq.2 and solved to obtain optimal solutions. The optimal solutions depend on the weights $\left(\omega_{k}\right)$ assigned to each fuzzy goal:

$$
\begin{aligned}
& \operatorname{Max} \sum_{k} \omega_{k}{ }^{*} \theta_{k} \\
& \theta_{k} \leq \mu_{Z_{k}} \\
& \theta_{k}, \mu_{Z_{k}}, \omega_{k} \in[0,1] \\
& \sum_{k} \omega_{k}=1
\end{aligned}
$$

where $\theta_{k}$ denotes the satisfaction degree of the kth objective (individual satisfaction degree of each objective).

\section{Numerical results}

In this paper, we analyzed an integrated productiondistribution of a multi-echelon dairy supply chain in Morocco. The manufacturer is a big company that has four geographically-dispersed production sites. Two different products are considered in this paper (pasteurized and UHT milk). Due to confidentiality issues, the real data has been modified and is randomly generated from distributions given in Table 1. $\mathrm{U}(\mathrm{a}, \mathrm{b})$ denotes uniform distribution between a and $b$. We define each membership function by defined upper and lower bounds of each objective function. The upper and lower bounds obtained by maximizing and minimizing each objective function separately are presented in Table 2 . The proposed MILP was solved on a $2.5 \mathrm{GHz}$ Intel Core i5 PC with 4 GB RAM using IBM ILOG CPLEX 12.4. The computational results are given in Table 3. The solution results is given in Table 4. It is clear that the value of weights influence on the relations between the different objectives functions. In Table $4,{ }^{\omega S L}$ and ${ }^{\omega T C}$ denotes the weights of service level and total costs objectives for each instance and the degree of satisfaction of the objective functions for the proposed method. 
Table 1. Random generation functions of parameters

\begin{tabular}{|c|c|}
\hline$D_{c, \text { pasteurized-milk,n}}$ & $1000 * \mathrm{U}(120,150)$ \\
\hline$D_{c, U H T-m i l k, n}$ & $1000 * \mathrm{U}(100,120)$ \\
\hline Critical rate & 066 \\
\hline$q q t_{\text {pasteurized-milk }}$ & 2 \\
\hline$q q t_{U H T-m i l k}$ & 30 \\
\hline Benefit $_{\text {pasteurized-milk }}$ & 200 \\
\hline Benefit $_{U H T-m i l k}$ & 200 \\
\hline$V P C_{p, s, j, t}$ & $\mathrm{U}(10,15)$ \\
\hline $\operatorname{Cap}_{s, j}^{\max }$ & $\mathrm{U}(18,20)$ \\
\hline$R t_{s, j, t}$ & $\mathrm{U}(10,16)$ \\
\hline$O T C_{s, j, t}$ & $\mathrm{U}(150,200)$ \\
\hline$I H C S_{p, s, t}$ & $\mathrm{U}(10,20)$ \\
\hline$I H C D_{p, s, t}$ & $\mathrm{U}(10,20)$ \\
\hline$S S S_{p, s, t}$ & $\mathrm{U}(600,650)$ \\
\hline$S C S_{p, s, t}$ & $\mathrm{U}(70,75)$ \\
\hline$T S C S_{p, s, t}$ & $\mathrm{U}(350000,400000)$ \\
\hline$S S A_{p, a, t}$ & $\mathrm{U}(600,650)$ \\
\hline$S C A_{p, a, t}$ & $\mathrm{U}(70,75)$ \\
\hline $\mathrm{T} S C A_{p, a, t}$ & $\mathrm{U}(15000,20000)$ \\
\hline$T C D_{s, a}-T C C_{a, c}$ & $\mathrm{U}(2,4)$ \\
\hline$T L T 1_{s, a}-T L T 2_{a, c}$ & $\mathrm{U}(0.1,0.2)$ \\
\hline$B C_{c, \text { pasteurized-milk, } n}$ & $\mathrm{U}(20,30)$ \\
\hline$B C_{c, U H T-m i l k, n}$ & $\mathrm{U}(15,20)$ \\
\hline
\end{tabular}

Table 2. Lower and upper bounds of the objectives

\begin{tabular}{|c|c|c|}
\hline Objectives & Lower bound & Upper bound \\
\hline Total costs & 2135890 & 2850070 \\
\hline Service level & $95 \%$ & $99 \%$ \\
\hline
\end{tabular}

Table 3. Computational results

\begin{tabular}{|c|c|}
\hline No of variables & 44303 \\
\hline No of integer variables & 18340 \\
\hline No of constraints & 56109 \\
\hline CPU (s) & 840 \\
\hline
\end{tabular}

Table 4. Solution results

\begin{tabular}{|l|l|l|l|l|}
\hline Instance & $\omega_{S L}$ & $\omega_{T C}$ & $\mu_{S L}$ & $\mu_{T C}$ \\
\hline 1 & 0.65 & 0.35 & 0.9175 & 0.8655 \\
\hline 2 & 0.20 & 0.80 & 0.8919 & 0.7760 \\
\hline 3 & 0.50 & 0.50 & 0.988 & 0.7655 \\
\hline 4 & 0.10 & 0.90 & 0.8851 & 0.8919 \\
\hline 5 & 0.90 & 0.10 & 0.9245 & 0.9036 \\
\hline
\end{tabular}

\section{Conclusion}

In this paper, we proposed a bi-objective mixed integer model to deal with the production-distribution problem found in the dairy supply chain in Morocco. The model aims to incorporate two objectives: the first objective function aims to maximize benefit by considering the shelf life-dependent pricing component and various cost components, such as setup costs, storage, distribution, and backorders. The second objective function aims to maximize the service level related to each product for customers. To deal with the imprecise preferences of the DM regarding the aspiration level of objectives. An approach based on Weighted Additive Fuzzy Goal Programming is used. The numerical experiments indicate that the proposed model is practical and tractable.

\section{References}

1. Erengüç, Ş Selçuk, Simpson, Natalie C, \& Vakharia, Asoo J. Integrated production/distribution planning in supply chains: An invited review. European Journal of Operational Research, 115(2), 219-236, (1999)

2. Chen, Zhi-Long. Integrated Production and Distribution Operations Handbook of quantitative supply chain analysis, 711-745, (2004).

3. Bilgen, Bilge, \& Ozkarahan, Irem. Strategic tactical and operational production-distribution models: a review. International Journal of Technology Management, 28(2), 151-171 (2004)

4. Bilgen, Bilge, \& Çelebi, Yelda. Integrated production scheduling and distribution planning in dairy supply chain by hybrid modelling. Annals of Operations Research, 211(1), 55-82, (2013)

5. Sel, C, Bilgen, B, Bloemhof-Ruwaard, JM, \& van der Vorst, JGAJ. Multi-bucket optimization for integrated planning and scheduling in the perishable dairy supply chain. Computers \& chemical engineering, 77, 59-73, (2015)

6. Lütke Entrup, Matthias, Günther, H-O, Van Beek, Paul, Grunow, Martin, \& Seiler, Thorben, MixedInteger Linear Programming approaches to shelf-lifeintegrated planning and scheduling in yoghurt production. International journal of production research, 43(23), 5071-5100, (2005)

7. Nakhla, M. Production control in the food processing industry: the need for flexibility in operations scheduling. International Journal of Operations \& Production Management, 15(8), 73-88, (1995)

8. Zadeh, L. A. Outline of a new approach to the analysis of complex systems and decision processes. Systems, Man and Cybernetics, IEEE Transactions on, (1), 28-44, (1973).

9. Tiwari, R. N., Dharmar, S., \& Rao, J. R. Fuzzy goal programming - an additive model. Fuzzy sets and systems, 24(1), 27-34, (1987)

10. Zimmermann, H. J. Fuzzy, set theory-and its applications. Springer Science \& Business Media, (2001) 